\title{
Gutachter(innen) 2009
}

Die Redaktion der PVS dankt allen Gutachterinnen und Gutachtern, die ihr bei der Auswahl der Beiträge im Jahr 2009 behilflich waren.

\begin{tabular}{|c|c|c|}
\hline Heidrun Abromeit & Klaus Jacob & Josef Schmid \\
\hline Björn Alpermann & Detlef Jahn & Susanne K. Schmidt \\
\hline Kai Arzheimer & Martin Jänicke & Hermann Schmitt \\
\hline Michael W. Baur & Viktoria Kaina & Kai-Uwe Schnapp \\
\hline Joachim Behnke & André Kaiser & Carsten Q. Schneider \\
\hline Marianne Beisheim & Ronja Kempin & Harald Schoen \\
\hline Heinrich Best & Markus Klein & Gunnar Folke Schuppert \\
\hline Harald Bluhm & Marianne Kneuer & Pascal Sciarini \\
\hline Tanja A. Börzel & Danko Knothe & Martin Seeleib-Kaiser \\
\hline Lothar Brock & Thomas König & Dieter Senghaas \\
\hline Manfred Brocker & Sabine Kuhlmann & Susumu Shikano \\
\hline Hubertus Buchstein & Hans-Joachim Lauth & Ulrich Sieberer \\
\hline Marius Busemeyer & Roland Lhotta & Tine Stein \\
\hline Aurel Croissant & Georg Lutz & Markus Steinbrecher \\
\hline Roland Czada & Hanns W. Maull & Barbara Steininger \\
\hline Nicolai Dose & Peter Mayer & Michael Stoiber \\
\hline Dieter Fuchs & Wolfgang Merkel & Christoph Strünck \\
\hline Klaus J. Gantzel & Ursula Münch & Andrej Stuchlík \\
\hline Thomas Gehring & Wolfgang Muno & Roland Sturm \\
\hline Axel Görlitz & Jürgen Neyer & Klaus Stüwe \\
\hline Stefan Gosepath & Franz U. Pappi & Petra Stykow \\
\hline Florian Grotz & Werner J. Patzelt & Jens Tenscher \\
\hline Christof Hartmann & Barbara Pfetsch & Paul W. Thurner \\
\hline Andreas Hasenclever & Susanne Pickel & Kerstin Völkl \\
\hline Anke Hassel & Heiko Pleines & Uwe Wagschal \\
\hline Hubert Heinelt & Thomas Plümper & Manfred Walther \\
\hline Tanja Hitzel-Cassagnes & Britta Rehder & Cornelia Weins \\
\hline Ursula Hoffmann-Lange & Robert Rohrscheider & Christoph Weller \\
\hline Everhard Holtmann & Edeltraud Roller & Christian Welzel \\
\hline Christoph Hönnige & Michael Ruck & Bernhard Weßels \\
\hline Sascha Huber & Thomas Saretzki & Bettina Westle \\
\hline Hartwig Hummel & Siegfrid Schieder & Joachim Wieland \\
\hline Ellen M. Immergut & Frank Schimmelfennig & Andreas Wüst \\
\hline Markus Jachtenfuchs & Utz Schliesky & Reimut Zohlnhöfer \\
\hline
\end{tabular}

